\title{
Produciendo el oasis, transfiriendo vulnerabilidades. Particularidades hidro-geomorfológicas y transformaciones socio productivas en el Valle de Uco, Mendoza, Argentina ${ }^{1}$
}

\author{
Producing the oasis, transferring vulnerabilities. Hydro- \\ geomorphological particularities and socio-productive \\ transformations in the Uco Valley, Mendoza, Argentina
}

Paula Mussetta ${ }^{2}$, Nicolás Parise Schneider ${ }^{3}$ y Jorge Ivars ${ }^{4}$

\begin{abstract}
RESUMEN
El proceso reciente de expansión del oasis del Valle del Uco hacia el piedemonte andino ha sido protagonizado por grandes emprendimientos agrícolas. Este avance modifica los cauces aluvionales de la zona y produce nuevas vulnerabilidades que impactan sobre otros actores y sectores del territorio. A partir de una metodología mixta entre técnicas cualitativas de investigación y un análisis propio de la geografía, analizamos cómo los rasgos del modelo agroproductivo vigente en la región se entrecruzan con las características hidro-geomorfológicas de este oasis; habilitando un proceso de producción y transferencia de nuevas vulnerabilidades cuenca abajo. Para explicar este proceso nos basamos en una conceptualización relacional de vulnerabilidad y en la lógica instrumental de producir la naturaleza propia de la acumulacion del capital. Nuestro análisis, aspira a hacer evidente un fenómeno que tiende a ser invisibilizado y resalta que el modelo agroproductivo sólo es exitoso para unos a costa de otros.
\end{abstract}

Palabras clave: vulnerabilidad, oasis, aluviones, transformaciones socio-productivas, Valle de Uco, Mendoza.

\section{ABSTRACT}

The recent process of expansion of the Uco Valley oasis towards the Andean foothills has been carried out by large agricultural enterprises. This advance modifies the alluvial channels of the area and produces new vulnerabilities that impact on other actors and sectors of the territory. Based on a mixed methodology between qualitative research techniques and a geographical analysis, we examine how the features of the dominant agricultural model in the region meet the hydro-geomorphological characteristics of this oasis, enabling a process of production and transfer of new vulnerabilities downstream. To explain this process we follow an approach based on a relational conceptualization of vulnerability and on the instrumental logic of producing the nature, typical of capital accumulation. Our analysis aims to make evident a phenomenon that tends to be invisible and highlights that the agricultural model is only successful for some at the expense of others.

Keywords: Vulnerability, oasis, floods, socio-productive transformations, Uco Valley, Mendoza. 
Entre los estudios de vulnerabilidad al Cambio Ambiental Global existe un extendido consenso en la necesidad buscar las causas de la vulnerabilidad en los modelos de desarrollo. Este consenso indica que los componentes de los modelos de desarrollo (paradigma tecnológico, reglas de los mercados, capital global) son mediadores de los impactos del cambio global y de esta forma aumentan la vulnerabilidad de algunos actores mientras disminuyen las vulnerabilidades de otros. La conceptualización más difundida que sintetiza esta idea es la de las dobles exposiciones (O’Brien \& Leichenko, 2010). Los trabajos derivados de esta matriz analítica nos enseñan que la vulnerabilidad al Cambio Global no sólo tiene múltiples causas (sociales, económicas y biofísicas) sino que dentro de esa multiplicidad, los factores no climáticos, como las condiciones del mercado, los procesos políticos, las crisis internas, suelen ser más relevantes que los eventos climáticos. Los factores no climáticos son llamados también segundas expocisiones. El entrelazamiento o superposición de vulnerabilidades climáticas y no climáticas en un mismo momento y sobre los mismos actores multiplica los impactos negativos, aumenta los riesgos y profundiza las vulnerabilidades. (O' Brien \& Leichenko, 2010). Estos estudios se esfuerzan por conocer las causas determinantes (evidentes y subyacentes) de la vulnerabilidad y las relaciones entre ellas. ${ }^{5}$

Algunas investigaciones que estudian los determinantes de la vulnerabilidad en contextos rurales en Mendoza encuentran que gran parte de las causas de la misma están vinculadas al desarrollo del agronegocio. ${ }^{6}$ Estos trabajos reafirman que para los productores rurales, eventos hidroclimáticos como sequía, granizo, heladas, exceso de lluvia, no son tan críticos como los problemas económicos; especialmente el de la brecha de precios entre los costos generales de la producción y el valor de los cultivos (Mussetta et al., 2018).

El estudio de la vulnerabilidad en el Valle de Uco que aquí desarrollamos constata estos hallazgos. Pero es un caso que aporta una particularidad respecto a otros contextos de la provincia de Mendoza. Nuestro caso permite ver que los grandes proyectos agrícolas no sólo configuran las segundas exposiciones sino que producen y transfieren nuevas vulnerabilidades. Éstas están asociadas a las formas de producir el oasis. En otras palabras, a los modos en que los nuevos emprendimientos productivos se instalan en las zonas más altas del Valle de Uco (sector superior de la Subcuenca del río Tunuyán) transformando el territorio a través de remoción de suelos, eliminación de cobertura vegetal, cambios de pendientes, colmatación y anulación de los cauces de wadis. Asimismo, y porque se asientan en lugares con alto riesgo aluvional, que a su vez ellos mismos contribuyen a aumentar, desarrollan prácticas de autoprotección que ocasionan daños severos en zonas más bajas. Como veremos, la hidro-geomorfología del lugar es fundamental en la explicación de este problema. Pero las nuevas vulnerabilidades no sólo se explican por ella sino que son consecuencia de las formas de apropiación del espacio, de la nueva producción del oasis y transferidas hacia otros actores, distintos a los que las producen.

\footnotetext{
La explicación de los determinantes de la vulnerabilidad es superadora de aquellas explicaciones influenciadas por el IPCC (Intergovernmental Panel on Climate Change) en las que la vulnerabilidad es el resultado último de la ecuación impacto/sensibilidad/capacidades de adaptación.

6 Recientemente, Larsimont en un estudio sobre el Valle de Uco (2018) definió al agronegocio vitivinícola como un modelo agropecuario que incluye grandes proyectos y engloba cinco elementos centrales: la desregulación económico-política, financiarización de la agricultura, se articula a un paradigma técnico-científico-informacional en tanto la construcción del espacio se articula en función de una tecnociencia informacional; en zonas secas el acaparamiento de tierras se da necesariamente ligado a espacios espacios verdes y con disponibilidad hídrica en el marco de esta flexibilidad en el acceso a los bienes comunes naturales; y finalmente, incluye una dialéctica producción-consumo particular en el que el consumo y la producción se construyen y gestionan mutuamente.
} 
Resaltando la dimensión relacional de la vulnerabilidad y una lógica instrumental de producción naturaleza, nos interesa evidenciar y desentrañar un fenómeno de producción y traslado de vulnerabilidades entre los nuevos actores agrícolas protagonistas de las transformaciones y los actores tradicionales del oasis. La naturaleza de este fenómeno está posibilitada por: las características hidro-geomorfológicas del Valle de Uco; la expansión desregulada de un modelo productivo a gran escala intensivo en capital y cuya producción está orientada al mercado externo y un estado aparentemente ausente en la planificación del uso del suelo pero expeditivo para permitir la gestión privada del territorio. La figura 1 grafica la relación entre estos componentes del problema de estudio. Nuestra propuesta de análisis está formulada para explicar exclusivamente el caso del oasis del Valle de Uco. No obstante, no se descarta la posibilidad de que el esquema pueda constituir una base para el estudio de otros casos producción y traslado de vulnerabilidades.

La metodología de análisis se basó en la triangulación entre distintos tipos de métodos. Por un lado, se utilizaron técnicas propias del método geográfico, que nos permiten analizar de manera conjunta los rasgos físico-naturales del territorio y las dinámicas socioeconómicas que se dan él (Santos, 2000). Por otro lado, la metodología del paradigma interpretativo de investigación (Vasilachis de Gialdino, 2013), fue fundamental para el análisis de la información primaria de carácter cualitativo generada a partir de entrevistas en profundidad (Kvale, 1996; Taylor \& Bogdan, 1992). Esta mirada interdisciplinaria es uno de los principales aportes de nuestra investigación.

El trabajo de campo consistió en dos etapas. En la primera (inicios de 2017) se realizaron un total de 30 entrevistas a productores con distinto grado de capitalización ${ }^{7}$ en la región del Valle de Uco. Las entrevistas indagaron sobre las percepciones de los actores sobre los cambios en el territorio, las exposiciones climáticas y no climáticas (respecto al uso del agua, heladas, granizo, nevadas, problemas económicos, políticos, sociales) y los impactos de ello en los modos de vida de las unidades productivas. El problema del impacto de los aluviones específicamente asociado a las prácticas productivas en las zonas más altas, fue un problema emergente del trabajo de campo. Fue así que en una segunda etapa (fines de 2017) se realizó una nueva ronda de 12 entrevistas a productores en los sitios en donde habíamos advertido este problema. En esta segunda etapa fue fundamental el uso de técnicas y conceptos propios de la geografía.

El texto está organizado de la siguiente manera. En primer lugar, desarrollamos una conceptualización sobre vulnerabilidad y sobre la producción capitalista de la naturaleza que se pone en juego en el análisis del fenómeno estudiado. Seguidamente, describimos las características hidro-geomorfológicas de la subcuenca y a continuación las transformaciones de la economía agroindustrial en el Valle de Uco. Estos dos apartados describen el marco de posibilidades de las nuevas formas de producción del oasis productora de vulnerabilidades. En el apartado siguiente bajo el título "produciendo el oasis" describimos cómo las prácticas y políticas hídricas, los vacíos en el ordenamiento territorial y la primacía de criterios particulares de los actores en el uso del suelo productivo van generando y trasladando las nuevas vulnerabilidades a las que nos referimos. Finalmente, concluimos que el modelo agroproductivo vigente es exitoso para algunos a costa de otros. El éxito del mismo se funda en la posibilidad de hacer un uso conveniente de los

La capitalización de los productores rurales es el principal criterio de la tipología propuesta por Manzur \& Carballo (2016), adoptada en el presente estudio Esta incluye: productores familiares sin capitalización; productores familiares capitalizados; productores capitalistas pequeños; productores capitalistas medianos y productores capitalistas grandes. 
bienes naturales y desarrollar una gestión privada de los riesgos naturales. Todo esto es promovido por los fundamentos y exigencias del mercado y el capital.

\section{Un marco para entender las vulnerabilidades}

La noción de que las acciones desarrolladas por unos actores en un lugar pueden transferir vulnerabilidades a otros actores $u$ otros sitios tiene una trayectoria dentro del campo de los estudios del Cambio Ambiental Global. En general, la literatura sobre vulnerabilidad desarrolla el concepto de "maladaptación" para abordar estos efectos o consecuencias no deseadas que podrían aumentar la vulnerabilidad de ciertos grupos, ya sea en el mismo lugar o en otro. Entre estos estudios, se destaca el trabajo de Eakin et al. (2009) que ofrece ejemplos del sector cafetero; el de Klein (et al., 2001) que utiliza un ejemplo de medidas de adaptación costera y el de Atteridge y Remling (2013) quienes rastrean las dimensiones sociales y económicas de los impactos de las adaptaciones del sector cafetero en Colombia. Incluso, con un caso similar al que desarrollamos en este trabajo, Adger et al. a través del concepto de "efectos espaciales indirectos" (spatial spillovers) demuestran cómo la construcción de nuevos terraplenes de inundación protegen a una comunidad mientras a la vez aumentan la vulnerabilidad de las comunidades cuenca abajo (Adger et al., 2005). La lógica de los "ciclos o bucles causales" (causal loops) también fue utilizada para estudiar cómo se vinculan las múltiples causas de la vulnerabilidad (Navarrete \& Gallopín, 2007). Estos aportes son valiosos porque destacan el carácter relacional de la vulnerabilidad que nos ayuda a interpretar nuestro objeto de estudio. Pero todos estos análisis se orientan a proponer instrumentos para mejorar la adaptación a las exposiciones climáticas y meteorológicas. Es decir, son estudios que permanecen dentro de los paradigmas de la adaptación. Nuestro análisis, se aleja de este marco porque entiende que, en condiciones en las que los grupos sociales experimentan los beneficios y los riesgos inherentes a las transformaciones sociales y ecológicas en formas muy desiguales, la adaptación sólo puede ser conservadora del statu quo de las inequidades (Watts, 2015). La adaptación así entendida contribuye a consolidar las diferencias de poder y de recursos derivadas de una inequitativa distribución de los riesgos y las recompensas en el marco de las relaciones sociedad-naturaleza.

La idea que la vulnerabilidad es producida y puede ser transferida destaca el carácter relacional de la misma y asume que las acciones de unos actores en un lugar podrían desencadenar cambios en el riesgo y la vulnerabilidad de otros actores en otros lugares. Lo especialmente problemático no es sólo que la vulnerabilidad se reproduce constantemente sino que algunos grupos reducen su vulnerabilidad a costa de la de otros. Este carácter desigual de la vulnerabilidad se agrava cuando sus impactos más nocivos recaen y se acumulan siempre sobre los mismos grupos. Y aquí encontramos otro argumento por el cual la noción de adaptación al cambio climático nos resulta problemática. Los sistemas adaptativos o resilientes suponen, a menudo, "órdenes socio-ecológicos altamente injustos porque las cualidades identificadas como resilientes son una función de relaciones de poder arraigadas que institucionalizan el desplazamiento del riesgo y la vulnerabilidad entre los grupos" (Taylor, 2015 :78).

La adaptación además, se rige por una lógica de la precaución que busca anticiparse a los riesgos y en última instancia aminorar o evitar impactos futuros (Amoore, 2013:9). De esta forma, los riesgos se vuelven calculables, pero esa calculabilidad es privada, es decir, son los actores 
individuales quienes tienen la responsabilidad de desarrollar prácticas de adaptación y ajuste al cambio climático.

Figura №1:

Esquema de producción y traslado de las vulnerabilidades.

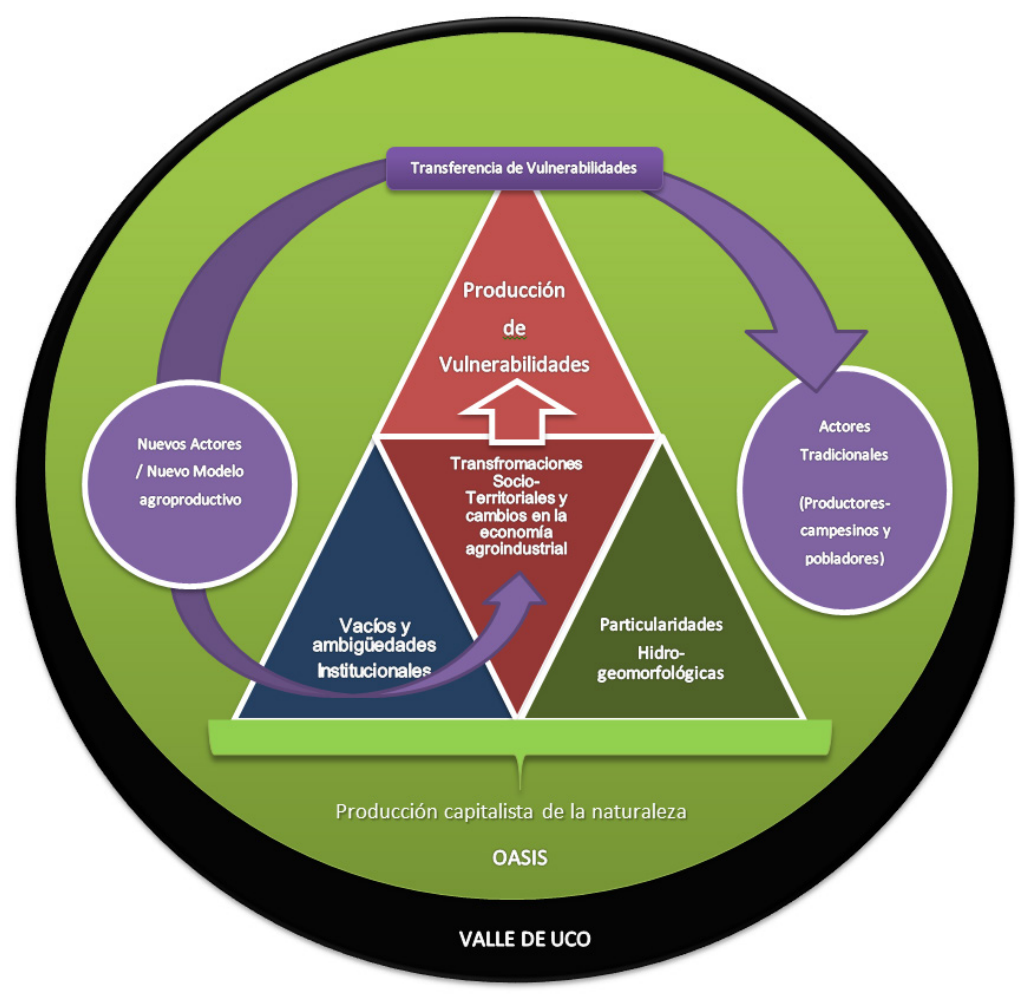

Fuente: Elaboración propia

\section{La producción capitalista de la naturaleza}

Otro conjunto de consideraciones que debemos tener en cuenta son las mediaciones que se establecen entre las transformaciones en la economía agroindustrial del Valle de Uco y las nuevas vulnerabilidades. Es necesario explicitar que en ellas se pone en juego una forma de producción capitalista de la naturaleza que se basa en una racionalidad instrumental.

En términos abstractos, podemos afirmar que los seres humanos entablan relaciones con el medio natural circundante y a la vez son parte de él. A menudo, este último hecho es soslayado en las sociedades capitalistas en la medida en que la naturaleza es conceptualizada como un objeto exterior a la sociedad y, sobre todo, como un objeto pasible de dominación. Ahora bien, estas relaciones sociedad-naturaleza, en la que la sociedad es parte y hacedora de ella, están mediada por diferentes formas de racionalidad. Ya Max Weber (1991) explicaba que occidente asistía 
(desde hace siglos) a un proceso de racionalización de la experiencia humana. Desde nuestra perspectiva, entendemos que este proceso tiene una dirección muy definida: se trata de un proceso específico de racionalización tendiente a un mayor grado de calculabilidad. A su vez, este proceso implica la difusión de un tipo particular de racionalidad, la racionalidad medios-fines o instrumental (Horkheimer, 1969). Esta racionalidad reflexiona sólo sobre los medios necesarios para alcanzar un fin; un fin que no es problematizado sino que queda subsumido a la esfera individual o a consideraciones éticas o morales, por tanto, por fuera de la reflexión científica.

Esta forma de razonamiento instrumental ha sido objeto de muchos debates y también de exaltación por parte de, por ejemplo, la economía neoclásica que reflexiona acerca del actor racional y su consecuente accionar. Idealmente, el empresario capitalista debe actuar de acuerdo a esta forma de racionalidad. El fin último de la empresa es la maximización de la ganancia y la minimización de costos o pérdidas. Este fin no es objeto de un debate profundo entre los empresarios. Lo que se debate es cuáles son los medios más adecuados para alcanzar ese fin. Esta forma de racionalidad empresaria, por tanto, procede de acuerdo a una abstracción tendiente a reducir la complejidad del medio sobre el cual se proyecta, en la medida en que tiene que clasificar las circunstancias en función de una representación binaria de ingresos y costos. Sin embargo, la acción humana individual es, inevitablemente, fragmentaria (Hinkelammert \& Mora, 2009). Esto significa que es muy difícil prever sus efectos no intencionales, no dependiendo estos de la buena o mala voluntad de los individuos sino de una gran masa de información inexistente o inalcanzable para un individuo o grupo. A pesar de ello, esta acción instrumental se halla inserta en una forma de organización social que la legitima como la forma de racionamiento más racional (valga la redundancia). En un sistema económico que impulsa la acumulación incesante y la externalización de costos (Wallerstein, 2004, 1995), es razonable que los empresarios se dejen llevar por esta forma de cálculo acotado, porque en última instancia es lo más racional para mantenerse como tales.

De este modo, es esperable que las relaciones, en particular las relaciones con la naturaleza, se establezcan mediadas por esta forma de razonamiento. En primera instancia considerar el medio como un objeto externo, y en segunda como algo que puede ser puesto al servicio del fin de la empresa. Las acciones empresarias estarían destinadas a instrumentalizar y dominar el espacio (y los eventos que lo constituyen) en función de objetivos previamente definidos, como la obtención de ganancia. En el caso que nos ocupa, el de los grandes emprendimientos agrícolas instalados en las partes altas del Valle de Uco, la ocupación de suelos de altitud, arenosos y profundos, con climas de marcada amplitud térmica, constituyen las bases ecológicas a partir las cuales obtener cosechas agrícolas prestigiadas en los mercados agro-alimentarios mundiales. Esto implica, en gran medida, eliminar la cobertura vegetal en zonas de alta pendiente que, en última instancia, absorben parte de las aguas que luego darían lugar a los aluviones. De este modo, la transferencia de vulnerabilidades hacia otros grupos resulta ser la consecuencia de la forma más eficiente de gestionar el uso del suelo. Aunque muy probablemente esto no sea percibido por los actores involucrados, las acciones tendientes a mitigar sus propios riesgos implican externalización de costos (es decir, reducción). Entonces, la capacidad autónoma de desviar cauces y/o cegarlos y autoabastecerce de aguas independientemente del sistema de administración superficial resultan ser "estrategias adaptativas" de estos actores empresarios que se ajustan a la lógica de razonamiento reseñada anteriormente. La apropiación y manejo de bienes comunes naturales (incluyendo las benéficas relaciones que se establecen entre ellos) aseguran su subsistencia como empresarios. 
Es desde este punto de vista que no podemos soslayar la dimensión relacional de los fenómenos sociales en el espacio. La vulnerabilidad constituye un fenómeno estrictamente social que se inserta como parte necesaria de un conjunto social complejo.

\section{Particularidades hidro-geomorfológicas del sector superior de la Subcuenca del río Tunuyán}

Para entender el proceso de producción de vulnerabilidades en nuestra área de estudio es preciso problematizar tanto la naturalización de los fenómenos sociales descriptos anteriormente así como tener en cuenta los condicionantes biofísicos implicados en el fenómeno estudiado. Esto significa que es importante considerar la dimensión biofísica del fenómeno aluvional. En el contexto del estudio de los impactos del Cambio Ambiental Global algunos fenómenos naturales que son potencialmente riesgosos, pueden ocurrir con mayor frecuencia e intensidad. Por ejemplo las tormentas convectivas estivales en el sector de llano y en los cordones montañosos al este de la cordillera frontal (Boninsegna, 2014).

Frente a estos cambios también resulta preciso señalar que nuestra área de estudio posee características hidro-geomorfológicas particulares en relación al sitio del oasis en comparación con otros oasis de la provincia de Mendoza. Esto supone que podemos considerarlo potencialmente más vulnerable a los posibles impactos de eventos aluvionales o escorrentías extraordinarias. Estas exposiciones pueden verse potenciadas por dos factores: por un lado, por lo ya dicho acerca de la mayor probabilidad de que se produzcan tormentas más intensas y más frecuentes; por otro lado por las transformaciones y expansión del oasis que pueden estar aumentando su vulnerabilidad frente a eventos aluvionales.

El oasis del Valle de Uco esta ubicado dentro de la denominada Cuenca del Tunuyán Superior que comprende la totalidad del Valle de Uco y a la cual en este trabajo nos referiremos como "sector superior de la Subcuenca del río Tunuyán". Se extiende al oeste sobre un sector de los Andes Centrales entre las denominadas cordillera principal y cordillera frontal. Es en esta zona donde se encuentran casi la totalidad de las nacientes de los arroyos que escurren luego sobre el área central de la subcuenca desde el piedemonte hacia el graben ${ }^{8}$. Sobre este último se extiende la mayor parte del oasis del valle, y es donde el río Tunuyán captura el resto de los arroyos en su escurrimiento hacia el noreste. Además, una pequeña franja longitudinal al este de éste sector de la subcuenca se encuentra sobre el sistema de huayquerías (Capitanelli, 1992), que cuenta con escasos y pequeños arroyos de régimen estacional que drenan de este a noroeste por una superficie accidentada a mayor altitud que la zona del graben.

El oasis del Valle de Uco se extiende desde el graben longitudinalmente hacia el oeste sobre el piedemonte de los cordones que conforman la cordillera frontal, aquí se presenta una de las primeras particularidades: a diferencia de los otros grandes oasis de la provincia, se encuentra

Larga depresión limitada en ambos lados por fallas normales paralelas, que dejan bloques elevados (horst) y entre los cuales el terreno se ha hundido por fuerzas internas. Se producen normalmente en un régimen tectónico distensivo y suelen estar asociadas a los primeros estadios de fracturación de placas y apertura oceánica. 
confinado entre accidentes orográficos. Por ello se trata de un oasis de valle y es, además, el oasis con mayor pendiente. Principalmente, el desarrollo de la vitivinicultura de altura impulsó la expansión del oasis sobre las tierras altas del piedemonte sobre los tres departamentos que lo conforman: Tupungato, Tunuyán y San Carlos.

El desarrollo del oasis sobre el piedemonte de la cordillera frontal constituye la primera particularidad. Esta unidad geomorfológica recibe más precipitaciones pluviales y nivales que la precordillera, principalmente por la influencia del anticiclón del Pacífico Sur. Sumado a esto hacia el sur del río Mendoza (que escurre en dirección oeste-este) no encontramos la denominada precordillera. La cordillera frontal entonces recibe, además (principalmente en época estival), precipitaciones pluviales producto de la exitación orográfica de las masas de aire húmedo que llegan desde el este por influencia del anticiclón del Atlántico Sur. Vale aclarar que al norte del río Mendoza también se presentan estas precipitaciones. Sin embargo, las mismas se localizan fundamentalmente sobre la zona precordillerana, ya que ésta se interpone con las masas de aire húmedo antes de que las mismas alcancen la cordillera frontal, captando así la mayor parte de las precipitaciones provenientes del océano Atlántico.

De este modo, a lo largo del Valle de Uco se extiende sobre el piedemonte una red dendrítica de ríos y arroyos (de origen principalmente nivo-glacial por fusión en la cordillera frontal y en la cordillera principal), siendo ésta la segunda particularidad de este oasis. Esta cuenca además contiene una compleja red de cauces secos y semipermanentes muy dinámicos que drenan las aguas de fusión de las nevadas y, principalmente, los excedentes pluviales que no se infiltran. Como ya explicamos, las lluvias estacionales se producen por excitación orográfica o convección por lo cual suelen presentarse de forma extraordinaria y localizada. Por ello, los cauces de los ríos y arroyos permanentes y no permanentes son muy dinámicos, los mismos pueden recibir los aportes de estas tormentas convectivas e incluso drenar aluviones. Es decir el Valle de Uco y particularmente su piedemonte presenta una intensa dinámica aluvional.

Como dijimos anteriormente, en este sector superior de la Subcuenca del río Tunuyán aún la red hídrica se encuentra en desarrollo y conforma un sistema hidrográfico dendrítico. Esto tiene una serie de ventajas en relación a la administración del agua, por ejemplo no fue necesaria la construcción de largos canales para la distribución de los caudales a lo largo del piedemonte del valle, sino que se han aprovechado los cauces naturales de los numerosos arroyos que descienden desde los Andes. Pero a su vez también pueden constituirse como un factor de mayor vulnerabilidad a las variaciones en los regímenes de los distintos ríos y arroyos que van descendiendo sobre el piedemonte. Por estas características resulta dificultoso la construcción de embalses dentro de este área.

Como podemos observar en la Figura 2 el oasis del Valle de Uco además de ser el único confinado entre accidentes orográficos (en franjas marrones), es el único que no cuenta con un embalse artificial de jerarquía para gestionar las variaciones anuales o interanuales de caudales (círculos azules). En la cartografía también se puede observar en líneas blancas el complejo sistema dendrítico de ríos y arroyos que van atravesando el oasis.

Otro elemento a considerar en relación a las particularidades es la pronunciada pendiente en la que se desarrolla este oasis. En una extensión estrecha (Entre 40 y 55 km) de oeste-este pre- 
senta pendientes de entre 1,6 y $2,4 \%$ que son comparativamente mucho más pronunciadas que en los oasis Norte y Sur de la provincia $(0,43 \%$ en el primero y 0,40 el segundo, midiendo las mismas entre los puntos a mayor y menor altitud sobre el nivel del mar). Esta pronunciada pendiente es otro elemento a considerar para la evaluación de la vulnerabilidad del oasis frente a escorrentías extraordinarias o aluviones.

Figura $\mathrm{N}^{\circ} 2$

Sistema Hidrogeomorfológico de Mendoza

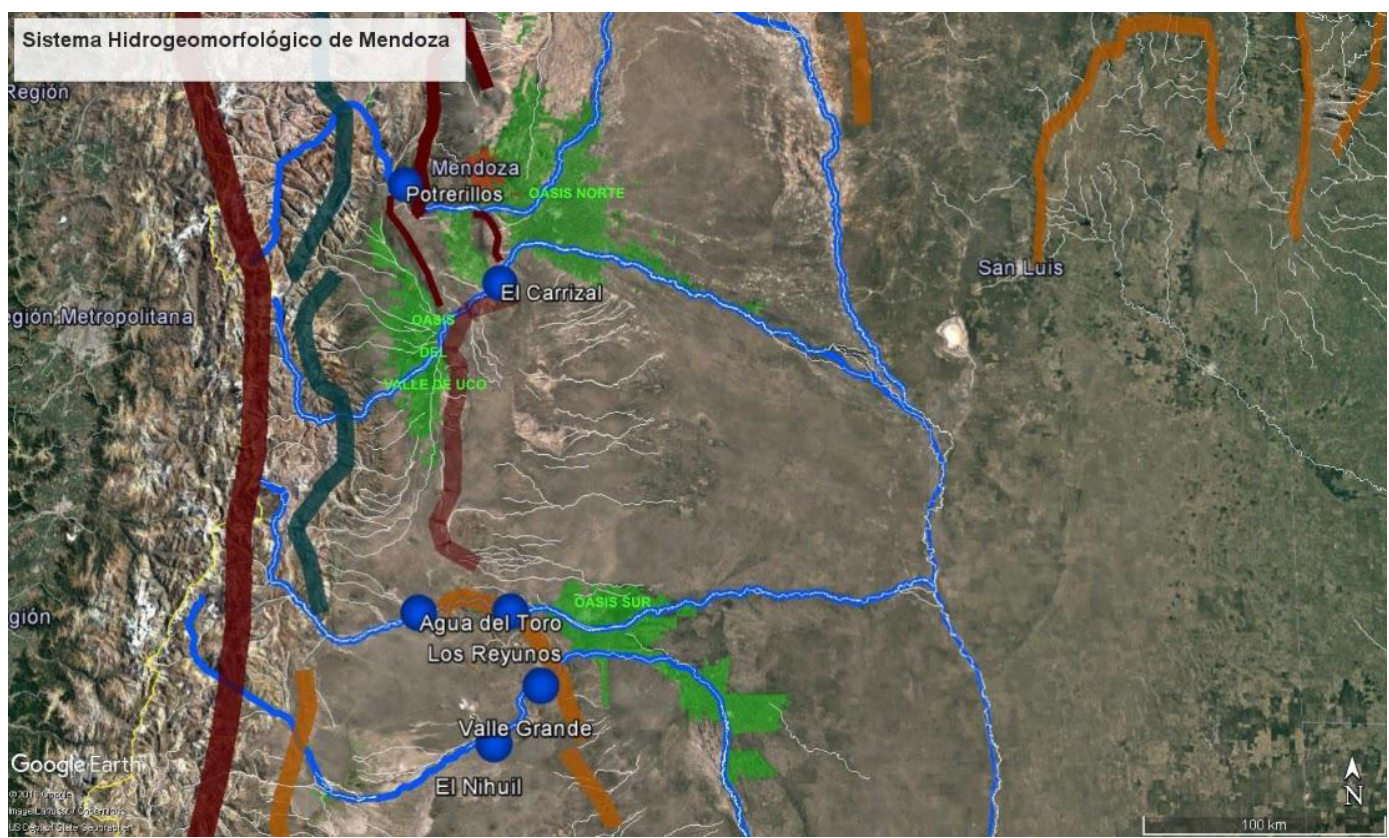

Fuente: Elaboración propia en base a "Google Earth"

En resumen, el Valle de Uco presenta particularidades hidro-geomorfológicas diferentes a la de los otros oasis provinciales. Por un lado, el oasis se extiende sobre una sola cuenca (la del río Tunuyán), mientras que los oasis Norte y Sur están localizados sobre dos cuencas, ríos Mendoza - Tunuyán y Atuel - Diamante, respectivamente. Mientras que otra particularidad, en términos hidrológicos, obedece a su configuración dendrítica; es decir, el oasis se localiza sobre una red de alimentación en donde se van realizando las capturas dentro del mismo. Por su parte, los otros dos grandes oasis provinciales se desarrollan en relación a su posición sobre las cuencas en sectores medios donde ya se ha superado la etapa de alimentación. De esta forma, la gestión hídrica en una y otra morfología es diferente. En los primeros el agua se gestiona jerárquicamente a partir de la entrada de un único cauce por cada cuenca, cada uno objeto de un manejo intensivo a través de un sistema de diques y embalses ubicados estratégicamente en sectores más altos (Potrerillos-Cipolletti en el río Mendoza; Carrizal en el río Tunuyán Inferior; Agua del Toro-Los Reyunos en el río Diamante y Los Nihuiles-Valle Grande en el río Atuel (ver figura 2). 


\section{Cambios en la economía agroindustrial del Valle de Uco}

El área bajo regadío del Valle de Uco comprende una superficie cultivada aproximada de 57.000 hectáreas (Registro de Uso de la Tierra del año 2014) de las 300.000 que ostenta la provincia (Van den Bosch \& Bocco, 2016). Este oasis presenta una trayectoria histórica distinta de la que siguieron el oasis norte y sur de la provincia. Podemos decir que el valle permaneció al margen de la dinámica agraria que caracterizó a la provincia durante el siglo XX. Mientras los oasis norte y sur se integraban plenamente al mercado nacional abasteciendo de vino la creciente demanda de la región pampeana que se encontraba en pleno auge agroexportador; el Valle de Uco, a la espera de la llegada del ferrocarril, declinaba con sus grandes estancias ganaderas históricamente destinadas al comercio de carne con Chile. De este modo, ocupó un lugar secundario en el plano provincial que mantuvo como su principal actividad agroindustrial la vitivinicultura. Durante este periodo, se desarrollaron en la zona cultivos frutihortícolas y un reducido número de agroindustrias para su procesamiento, ambos destinados al abastecimiento del mercado interno (Larsimont et al,. 2018).

Hacia la década de 1990, esta zona fue objeto de una fuerte transformación. El valle recibió un gran flujo de inversiones en coincidencia con las oportunidades que ofrecían las políticas de apertura y desregulación económica características de estos años, especialmente vinculadas a la "nueva vitivinicultura" (Montaña, 2008). Las excelentes cualidades climáticas y edafológicas de este piedemonte y la posibilidad de adquirir (por distintos medios) grandes extensiones de tierra y, sobre todo, acceso a aguas subterráneas para el riego de estas extensiones propiciaron el desembarco de grandes inversiones (Larsimont, 2018). Huelga aclarar acá que las condiciones agroecológicas del valle fueron muy apreciadas por los capitales que arribaron a la provincia. En palabras de Armando Llop, el río "Tunuyán superior es la panacea de las cuencas" porque posee gran cantidad de arroyos, zonas de recarga y las aguas más prístinas y claras de la provincia. En este sentido, el director del Centro de Economía, Legislación y Administración del Agua (CELA) explica que la cuenca tiene excelentes condiciones y por eso se avanzó mucho en la explotación de acuíferos: "obviamente los particulares se dieron cuenta a tiempo, porque esta es una de las zonas en donde más se avanzó en la explotación de aguas subterráneas en los últimos quince o veinte años" (Nota de prensa, 2015).

Este proceso de transformación reconfiguró toda la economía agroindustrial del llamado Oasis Centro. En los tres departamentos que componen el valle, el cultivo predominante es la vid y en variedades de alta calidad enológica. Esta especialización se dio en paralelo a un importante proceso de concentración de la propiedad. ${ }^{9}$ La dinámica intercensal de los últimos 30 años muestra la creciente preeminencia de este proceso. “En 1988 la mitad de los productores cultivaba menos de 4 has. y en la actualidad ese valor casi alcanza las 7 has. Se atribuye este crecimiento al desarrollo vitícola de los distritos altos y a una reducción simultánea de las fincas de las zonas más bajas, que fueron históricamente de menores dimensiones" (Van den Bosch \& Bocco, 2016: 154).

\footnotetext{
La transformación en el Valle de Uco disparó el precio de la tierra en general con distintos impactos para la población local. Por un lado, en las áreas cercanas a los centros urbanos se inició un proceso, contrario a la concentración, de subdivisión de las parcelas generando un sistema urbano rural o urbano disperso con casas de fin de semana u otras actividades donde se pierden las condiciones propias de la ruralidad para producir. (Barrientos, 2013: 177)
} 
Estas transformaciones son relatadas por los productores y trabajadores rurales: los dueños cambian. En la parte del corredor del vino son todos de afuera. Acá abajo los que antes hacían chacra se dedican casi todos a la uva porque es más rentable. Calculo que algún día se frenará el precio porque va a haber muchísima uva. Antes acá se hacía mucho ajo. También muchos han arrancado frutales para meter uva. Y se han dedicado de lleno a la uva. Yo toda la vida he hecho chacra, pero desde el año que viene voy a hacer uva y tengo que aprender todo lo que es el trabajo de la uva. Contratamos gente pero también tengo que aprender. El cambio de los cultivos te lleva a eso. (Comunicaciones Personales, obtenidas entre julio y diciembre de 2017)

Aunque de forma menos evidente, también en la horticultura operó un proceso de concentración. "Los horticultores diversificados se mantuvieron estables en superficie, pero se han concentrado, ya que se redujo el número de productores" (Van den Bosch \& Bocco, 2016: 158). Además podemos apreciar un proceso de desplazamiento de la frontera agropecuaria hacia el oeste, a zonas más altas y más propicias para la implantación de vides destinadas a exigentes mercados internacionales.

El elemento central que explica este proceso es el desembarco de grandes inversiones de capitales foráneos (nacionales y extranjeros) en emprendimientos agroindustriales orientados fundamentalmente a los circuitos agroalimentarios globales. La preponderancia del modelo tiene también un correlato en las relaciones sociales. Los habitantes locales son espectadores de estos cambios que se imponen sin mejoras evidentes y de largo plazo para ellos. La mayoría pondera de manera positiva los cambios que trae el modelo, porque trae cambios tecnológicos pero reconocen lo efímero de los beneficios para quienes estaban allí previamente. "Lo que ha servido de las empresas de afuera es el tema de que hay muchísimo trabajo para la poda y levantada. Pero es limitado porque el que sabe podar tiene trabajo en invierno y nada más." (Productor Rural, Comunicación Personal, Octubre 2017) "No es que no quiero que vengan (los extranjeros), si traen tecnología, traen el bienestar, dan trabajo, pero ellos tienen un dinero que es mucho más fuerte que el mío ¿Cuál hubiera sido mi sueño? Que todas estas grandes inversiones hubieran dejado una buena escuela y buen centro de salud, se encuentran en la zona más cara de Mendoza, en cuanto al turismo del vino, pero no hay escuelas secundarias de calidad en esta altura, ni siquiera pensar en escuelas técnicas. O sea si vos me dijeras: “¿Estas desconforme?" No, no estoy desconforme, me hubiera gustado que hubieran más argentinos, pero vamos a ser claros, en el año 90 una hectárea acá valía 100 dólares; en el 93, 300 dólares en el 96, 3.000 dólares y hoy día no conseguís una hectárea en menos de 80.000 dólares con agua y plantada" (Productora Rural, Comunicación Personal Octubre 2017). Y si bien estas también son vulnerabilidades propias del nuevo modelo agroproductivo, nuestro análisis se enfoca en el conjunto de aspectos en el que la racionalidad instrumental que rige dicho modelo se entrelaza con las condiciones hidro-geomorfológicas del lugar. En el siguiente apartado, nos detenemos a explicar estas relaciones.

\section{Produciendo el oasis, transfiriendo vulnerabilidad}

La instalación de estos emprendimientos reconfigura la hidrogeomorfología del lugar: grandes remociones de tierras que producen cambios de pendientes, colmatación de wadis, desvío de arroyos, o de cursos no permanentes, desagües borrados o cultivados. Todo ello no exime a estos nuevos grandes actores a ser impactados por las escorrentías extraordinarias. Por ello, se 
protegen y construyen sus propias defensas aluvionales con el objetivo de desviar el agua según la conveniencia del que hace el desagüe y no hacia donde tiene que ir el agua (Figura 3). Así la forma en que estos emprendimientos ocupan y producen el territorio del oasis, con las características propias de la racionalidad instrumental, tienen la posibilidad de controlar en su beneficio la morfología de escorrentía propia del terreno. Pero no es posible hacerlo sin consecuencias para otros. "Antes ahí no había nada, pero luego empezaron a sembrar álamos y hacer chacras y fueron cortando y bueno.. porque no querían que el agua fuera por las fincas de ellos así que hacían unos zanjones por la orilla del camino y el agua tomaba por ahí y a medida que iba pasando la creciente iba haciendo destrozos" (Productor Rural, Comunicación Personal, octubre 2017) El control de los riesgos, sólo es posible a expensas de otros. "Levantan estos terraplenes sin tener en cuenta el impacto social, ambiental y económico que producen en el territorio más abajo. Los cauces se desbordan y tenemos inundaciones aguas abajo en distritos como Vista Flores". (Barrientos, 2013: 177) "Las fincas hacen terraplenes y esto se ve en los mapas satelitales o desde la ruta. Subiendo al Manzano, las fincas mirando hacia el sur hicieron terraplenes de $6 \mathrm{~km}$ y nadie controla nada. Es lo mismo del corredor del vino. Si en cada puente de esa ruta te paras, y miras para los dos lados vas a ver que arriba del puente achicaron el canal, plantaron viña hasta adentro del cauce, dejaron un cauce chiquito y algunos le pusieron álamo para retener un poco más y si saltás del otro lado, queda el socavón. Y del otro lado del corredor productivo cada vez que llueve se hace una carca enorme, viene vialidad (Vialidad Nacional) y lo tapa de nuevo". (Productor vitícola, Comunicación Personal, septiembre 2017). Estos relatos evidencian cabalmente cómo estas nueva forma de producción del oasis crean vulnerabilidades y las transfieren de unos actores a otros.

Los efectos se notan en zonas cultivadas especialmente con las tormentas fuertes de verano. "Venía el agua por el medio del callejón y esa agua partía al medio la finca. La creciente venía por el arroyo y por arriba y quedabas aislado, no salías. Por este problema el patrón perdió una hectárea de ajo chino, entera la hectárea. Porque un poco que arrastraba y otro poco lo tapó de barro" (Encargado finca hortícola, Comunicación Personal, septiembre 2017).

Ante estos eventos, los productores afectados no consiguen resolver los daños y mucho menos modificar las condiciones que los originan: "La creciente esa que se va por la calle termina quedando en las fincas de abajo" ... "pero además con lo perdido, no hay remedio. Cuando nos agarró la crecida hemos perdido dos corridas de ajo y lo hemos tenido que dejar ahí. Y no lo podes vender ni para semilla porque si se te mojó el ajo tratado no te sirve para semilla." (Encargado de finca, Comunicación Personal, octubre 2017). En otro tramo de la entrevista este mismo productor afirma que "fuimos a quejarnos varias veces para que Hidráulica y Vialidad, nos abrieran el camino y para que le hiciera un desagüe como tenía que ser porque esa agua iba tomando por las fincas para llegar al arroyo. Pero nunca lo hicieron (...) por ejemplo, la finca de ahí al lado lo que hizo fue hacer un zanjón grande donde va la calle pública y esa tierra la hizo un terraplén. Entonces la creciente que baja, choca en el terraplén y sí o sí se va por la calle. Es la única manera. Entonces o todos tendrían que hacerse un terraplén o Hidráulica hacer un buen zanjón para que el agua vaya directo al arroyo. Si no, no hay manera. Y esas partes quedaron aisladas, esos años del fenómeno del niño la calle iba por al lado del arroyo y se cayó la mitad de la calle. Se desmoronó. Y los chicos estuvieron dos semanas sin ir a la escuela porque el micro no podía pasar por ahí porque por arriba se inunda todo. Así que no salis ni por el arroyo ni por la calle de arriba de Capiz." (Encargado finca, Comunicación Personal, octubre 2017). 
Figura $\mathrm{N}^{\circ} 3$

Dinámica erosiva en los márgenes del denominado "corredor productivo" ruta provincial n89 Tupungato, Valle de Uco

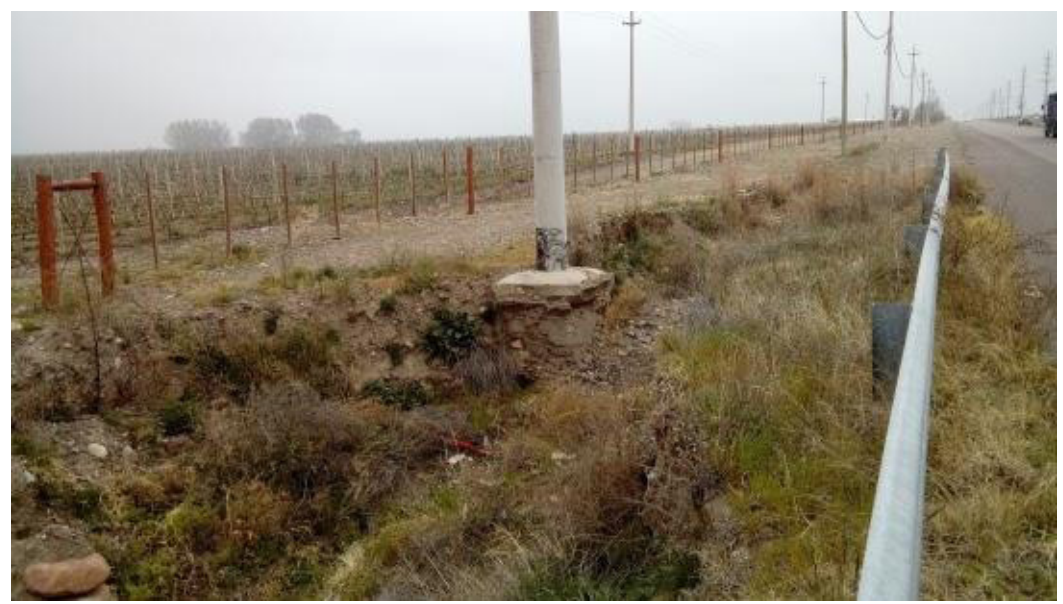

Fuente: Fotografías capturadas por el equipo en octubre de 2017

\section{Prácticas de manejo de las tierras cultivadas}

Un aspecto central de la nueva producción del oasis es el tipo de prácticas de manejo de estas nuevas y extensas unidades productivas. La expansión de estos capitales como ya dijimos, no sólo se realizó sobre áreas previamente cultivadas en reemplazo de otros cultivos con menores posibilidades económicas, sino que la expansión se produjo de manera especial sobre campos incultos y vírgenes, periféricos al oasis cultivado. Todo ello avanzó sin una planificación pública del uso del suelo y sin una visión de la integralidad del territorio. Las modalidades del uso del suelo estuvieron encabezadas por la iniciativa de los propios inversores. Los criterios que guiaron la instalación de los nuevos emprendimientos fueron los de los ingenieros agrónomos o profesionales que asesoraban a los inversionistas en función de los objetivos propios de la empresa. En las figuras 4 y 5 se puede apreciar como luce el paisaje actual en el Valle de Uco.

De este modo, en el Valle de Uco asistimos a una homogeneización del paisaje agrícola debido a la preponderancia de la vid junto a la utilización de criterios de diseño de fincas totalmente dispares y desvinculados entre sí. Todo ello ha incrementado peligrosamente los riesgos de aluviones e inundaciones en poblaciones y fincas aguas abajo. (Portella et al., 2015: 2) "Todo se hizo con una óptica nula en ecología y casi $100 \%$ ingenieril, muy cuadrado, líneas rectas mirando al mercado principalmente. Algunos querían la perfección y en eso tapaban desagües naturales. La estructura de estos viñedos consiste principalmente en espalderos que van cortando la pendiente principal, pero en realidad en el lugar hay múltiples pendientes, no una sola". (Ingeniero Agrónomo, Comunicación Personal, 4 noviembre 2017). Además, es importante mencionar que la disposición interfilar de los viñedos hace caso omiso de la pendiente del terreno, por el contrario el diseño está sólo en función de la exposición solar del viñedo que favorecerá no sólo la concentración de azúcar de la uva sino otras características organolépticas y, por tanto, la calidad enológica del vino a colocar en el mercado mundial. 
Figura $\mathrm{N}^{\circ} 4$

Viñedo en altura, Departamento Tunuyán, Valle de Uco

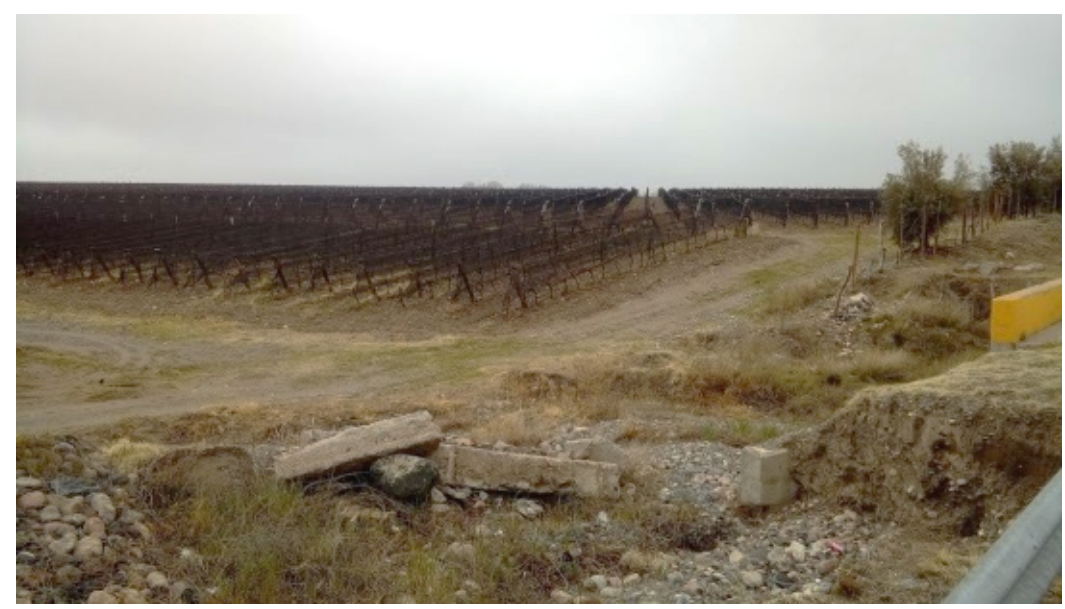

Fuente: Fotografías capturadas por el equipo en 2017

Los aspectos agronómicos asociados a estos nuevos usos del suelo se mezclan con las características físicas del lugar. La racionalidad instrumental ignora la geomorfología y el éxito de su consecución reside en la transferencia de las externalidades hacia otros. Como explica un experto entrevistado, "previo a la instalación de estos emprendimientos, el campo natural que antes estaba en estos sitios, tenía por diversos medios, la posibilidad de ayudar a retener el agua que escurría y a infiltrarla. Actualmente, la falta de cobertura en el suelo y vegetación de raíces profundas, facilita el escurrimiento y obstaculiza la infiltración". (Ingeniero Agrónomo, Comunicación Personal, 12 octubre 2017). La modificación de la cobertura de flora natural del suelo y en consecuencia de las condiciones de infiltración generaron un claro aumento de la erosión hídrica. La vegetación nativa fue reemplazada por viña, cuyas raíces son más superficiales que las raíces de la vegetación desmontada. Esto en parte es consecuencia de las prácticas de riego. "Esto a su vez se ve favorecido por el patrón de uso del riego que genera la concentración de raíces en áreas más superficiales, porque se riega lo necesario, lo mínimo que necesita el cultivo. Entonces no se riegan plantas acompañantes ni se deja que el riego se infiltre. El agua queda ahí en la superficie prácticamente. Los cultivos de vid generan una estructura del subsuelo muy homogénea y poco profunda en la distribución de raíces". (Ingeniero Agrónomo, Comunicación Personal, 4 noviembre 2017).

Además de esto, otra característica del diseño es la poca vegetación existente. Existe una tendencia muy extendida a mantener estos espacios limpios. En un estilo de producción de campo limpio entre hileras, los espacios interfilares van acompañando la pendiente sin nada que los cubra. Ello contribuye al cambio en la dinámica del agua.

Este problema no pasa inadvertido a los pobladores, quienes identifican con claridad su origen. "...Lo que pasa es que al ocupar los campos nuevos, las fincas, viste que el agua tiene su cauce y cuando empiezan a trabajar las rellenan. Entonces el agua tiene que tomar por algún lado. Entonces como cerraban la salida para el arroyo, tomaba por la calle porque la calle quedaba en un 
bajo y por ahí se iba desparramando por las otras fincas que estaban más en el bajo. La calle era como el cauce. Y a nosotros nos cubría la mitad de la finca." (Encargado de Finca, Comunicación Personal septiembre 2017).

Figura №5

Viñedo en altura, Departamento Tupungato, Valle de Uco

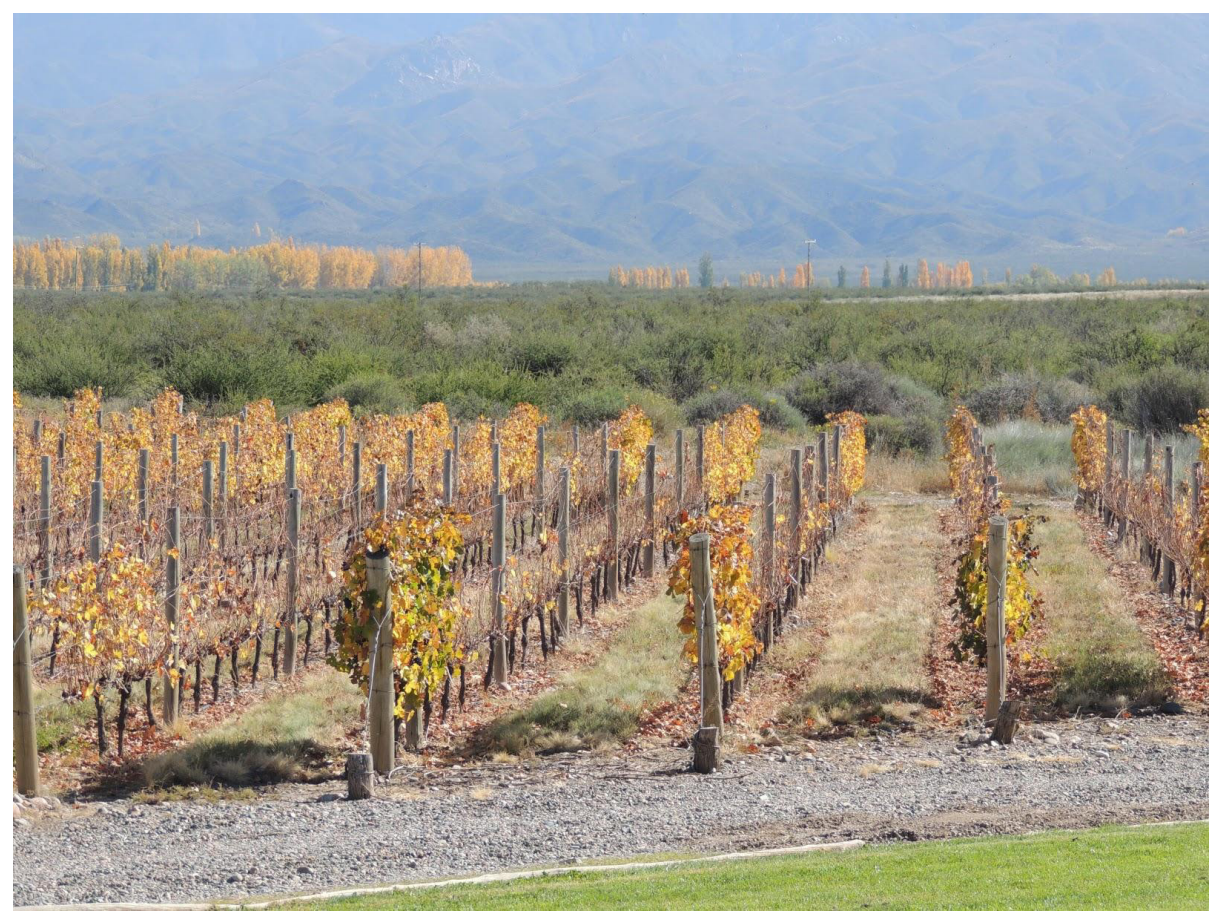

Fuente: Fotografías capturadas por el equipo en 2017

Las instituciones locales tampoco son ajenas. Por ejemplo, este problema fue explícitamente resaltado en los Diagnósticos que se desarrollaron como parte de la elaboración de los Planes de Ordenamiento Territorial en los municipios del Valle de Uco. La finalidad de tales diagnósticos era justamente identificar problemas que luego la planificación territorial municipal debía "corregir". "Hay firmas que han borrado canales aluvionales de siglos de formación, que han sido formados en épocas glaciares pero luego está la fuerza impulsora...el agua busca el gradiente que le parece más favorable.. y es por donde se va a conducir" (Barrientos, 2013: 135) (Figura 6). Si bien esa planificación integral guiada por el municipio todavía está en discusión cinco años después, también es notable la ausencia de intervención (ex ante y ex post) de las instituciones públicas directamente relacionadas en este tipo de asuntos (por ejemplo, de las Direcciones de Vialidad y de Hidráulica). "Esos zanjones más que nada los hacen los propietarios. Porque las calles estaban como abandonadas y entonces las mismas empresas son las que abren las calles para llegar a sus fincas. El resto las tenía que abrir Vialidad y nunca las abría. En la parte que nosotros estábamos nunca las abrieron. Nosotros para llegar a la finca pedíamos permiso a la finca de al lado que daba a la calle" (Productor Frutícola, Comunicación Personal septiembre 2017). Este vacío que dejan las instituciones en las orientaciones para el uso de suelos rurales, es llenado por las empresas, con sus criterios, con su racionalidad. 
Figura № 6

Wadi cultivado y ocupado en finca de viña de Tupungato, Mendoza

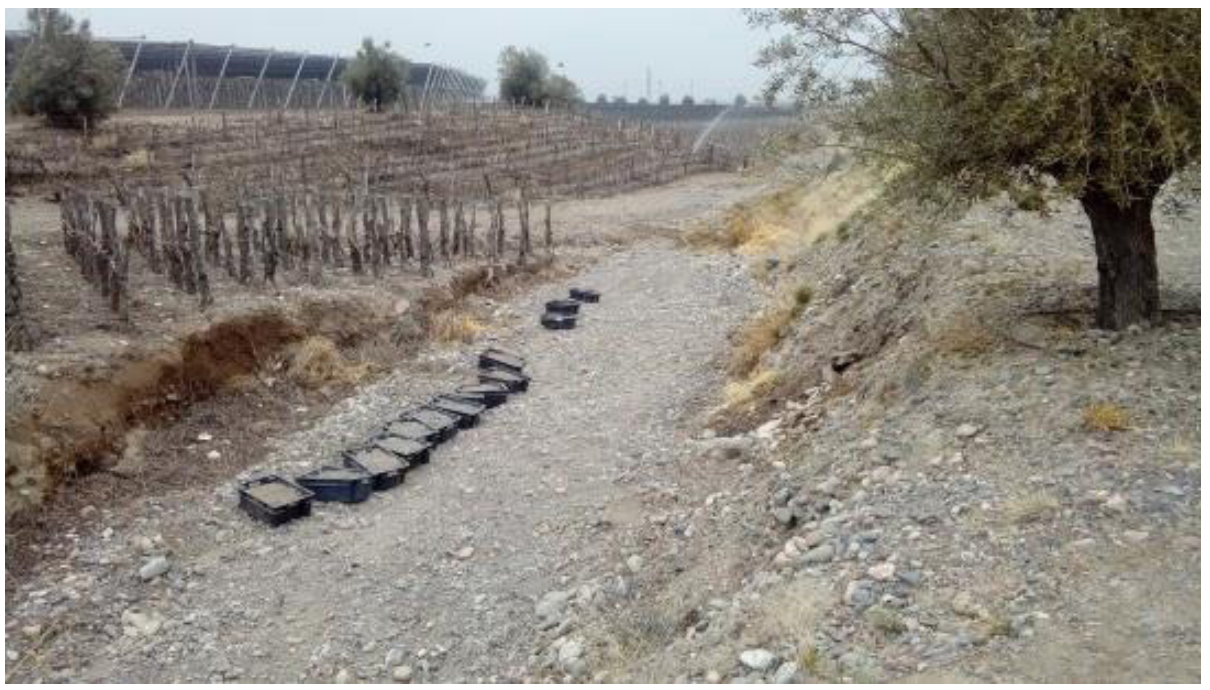

Fuente: Fotografías capturadas por el equipo en octubre de 2017

\section{La institucionalidad del agua y el riego}

En esta relación entre nuevas modalidades de producción del oasis y creación de nuevas vulnerabilidades, el agua de riego es un elemento central. Por ello, no se puede pasar por alto el papel que tuvo la gestión del agua, y en particular del agua subterránea en hacer posibles estas formas de producción del oasis. Tradicionalmente, como se mencionó más arriba, el oasis del Valle de Uco se distinguió como una región frutí-hortícola, con presencia de pequeñas y medianas unidades productivas. Estas explotaciones aprovechaban, fundamentalmente, las aguas provenientes de los arroyos y ríos de origen nivo-glacial. Hacia fines del siglo XX el inicio de la expansión de la inversión en grandes proyectos agroindustriales (fundamentalmente bodegas) descrito en el apartado anterior, se desarrolló hacia las márgenes del oasis tradicional de riego superficial y mediante el bombeo de aguas subterráneas (Larsimont, 2018). Estas perforaciones sólo son aprovechables con la incorporación de tecnología de riego, concretamente sistemas presurizados como el goteo. Es decir, esta transformación del territorio productivo del Valle de Uco no hubiese sido posible sin el acompañamiento de la política hídrica y la voluntad de su autoridad.

En ese plano institucional, en primer lugar, es fundamental la fragmentación existente entre la gestión superficial y subterránea del agua, rasgo propio e histórico de la institucionalidad del agua en Mendoza. Como ejemplo, basta mencionar que el agua subterránea se regula a partir de la asignación de licencias de perforación según especificaciones flexibles con criterios de diverso grado de (in)formalidad y el poder de la concesión recae sobre el Tribunal Administrativo del Departamento General de Irrigación. La diferencia con la gestión del agua superficial es notable cuando se advierte que se requiere una ley y la aprobación de más de la mitad de los miembros de la Legislatura Provincial para otorgar nuevos derechos superficiales. Es decir, la flexibilidad en la gestión de agua subterránea es histórica y no un rasgo propio del período de expansión del agronegocio. Pensar cómo se hubiese desarrollado este proceso con un sistema rígido de otorga- 
miento de permisos de perforación sería especular. Pero sí se puede afirmar que esta flexibilidad está asociada al margen de maniobra que las empresas requieren, y que es justamente un rasgo definitorio de este modelo (Larsimont, 2018).

Además de esta condición de la estructura normativa, un segundo aspecto del acompañamiento de la autoridad hídrica se advierte en prácticas políticas mucho más coyunturales y concretas. La expansión de la frontera cultivada a las zonas del piedemonte estuvo acompañada por la autoridad del agua mediante el otorgamiento de permisos de perforación. Según datos del Departamento General de Irrigación (DGI) en el Valle de Uco, la ubicación espacial de las perforaciones corrobora que los pozos más nuevos son los que más al oeste se encuentran y están por encima de los $1000 \mathrm{msnm}$. para lograr mayor amplitud térmica y elaborar vinos de alta gama (DGI, 2016: 317). Otro ejemplo es la política de sustitución de pozos. Esta consistía en que para obtener una nueva licencia de perforación los regantes debían presentar a cambio otra licencia de un pozo que por diversos motivos ya no fuese explotado (Mussetta et al., 2018).10

Ante esta posibilidad de extender la frontera agrícola hacia mayores altitudes mediante el uso de agua, los estudios elaborados por la institución a cargo de la política hídrica alertaban que "si bien, ninguno de los tres departamentos (del Valle) se encuentra dentro de la Declaración de Prohibición de construcción de nuevas perforaciones, sí es procedente declarar esta restricción" (DGI, 2016: 228). Al mismo tiempo, entendían que la vigencia de las restricciones dependería fundamentalmente de los estudios de acuíferos en elaboración y de las disponibilidades hídricas (oferta y demanda). La autoridad hídrica advertía también que las malas prácticas agrícolas en las zonas altas habían generado aluviones e inundaciones en la partes bajas como en Vista Flores (DGI, 2016 : 134). No obstante esas advertencias, la política hídrica ha demostrado un accionar errático y ambiguo ya que por un lado se alarma por las consecuencias de la expansión agrícola pero por otro habilita el uso ilimitado de aguas subterránea. Es claro que los nuevos emprendimientos encontraron en la política hídrica el apoyo y acompañamiento necesario para estas nueva modalidades de producción del oasis. Claramente, el DGI de manera directa o indirecta fue acompañando la consolidación de estas transformaciones.

Esta ambigüedad en el posicionamiento del DGI también se expresa en la definición de las definiciones de eficiencia en el riego que la institución realizaba. En los Balances Hídricos recientemente elaborados encontramos que la Ley 1920 de Cultivos Clandestinos considera ilegítimos a los cultivos realizados con aguas públicas en inmuebles de mayor superficie a la expresada en la concesión de riego o mayor al derecho legalmente empadronado en los registros oficiales. A su vez, desconoce y condena a quien a través del uso eficiente del recurso hídrico que legalmente le corresponde amplíe su frontera productiva (DGI, 2016: 179). El mismo DGI que indica que esta situación resulta insostenible a la luz de las exigencias actuales en el mismo estudio, páginas seguidas, propone ampliar la zona cultivada pero a partir de la exigencia del uso eficiente del agua, definiendo la eficiencia como razonable, es decir, aquella eficiencia que pueda alcanzarse con un buen mantenimiento y administración del recurso hídrico (DGI, 2016: 197).

10 Esto disparó la compra de tierras de menor calidad para cultivos en zonas no aptas para uso agrícola sólo para hacerse de las licencias para canjearlas por otras en las mejores zonas. Otra práctica usual de los grandes productores, fue la compra de fincas con perforaciones asignadas con el único fin de bombear el agua a las mejores tierras tierras de mayor altitud, fuera del oasis. 


\section{Conclusiones}

En el trabajo analizamos una problemática social como la vulnerabilidad de algunos pequeños y medianos productores rurales y pobladores del Valle de Uco en Mendoza frente a los efectos de escorrentías extraordinarias y aluviones. Para la descripción y análisis del problema se tuvieron en cuenta no sólo los factores sociales intervinientes sino también las características hidro-geomorfológicas del lugar que aumentan la vulnerabilidad general del valle. Explicamos que las causas del fenómeno están vinculadas a esas características biofísicas pero también a una nueva forma de producir el oasis que adscribe a la lógica de acumulación de grandes proyectos agrícolas. A partir de consideraciones muy abstractas como la racionalidad subyacente al accionar empresario y la comprensión de los aspectos relacionales de la vulnerabilidad, caracterizamos un fenómeno concreto que se constituye en un espacio socio-natural con unos aspectos biofísicos propios que son fundamentales para dar cuenta del fenómeno.

De acuerdo a lo sostenido hasta el momento, podemos aseverar que las nuevas formas de producir oasis no son inocuas dado que la acción social es fragmentaria y no es posible prever cabalmente sus efectos indirectos porque se lleva a cabo en un medio socio-natural incierto. Al mismo tiempo, estas formas de producción del espacio-oasis se basan en la capacidad que tienen los grandes actores que protagonizan estas transformaciones de incidir sobre el espacio más allá de sus propias explotaciones y de trasladar los costos y las exposiciones a productores y actores no directamente vinculados a ellos. Las motivaciones de estos grandes actores para acceder a napas de excelente aptitud hídrica implica la movilización de recursos para acceder a bienes naturales altamente valorados y reconocidos, no solo económica sino también simbólicamente en los mercados estandarizados internacionales. No obstante, la acción instrumental fragmentaria tiende a soslayar el hecho de que el agua es un bien muy susceptible de ser afectado. Pero además, en un marco de incertidumbre reinante, es incapaz de identificar plenamente a los potenciales actores perjudicados por estas formas uso del agua y el suelo irrigado.

Asimismo, otra condición de posibilidad de ocurrencia de los fenómenos aquí analizados están vinculados al accionar pendular de las instituciones estatales entre los vacíos de regulación y las condescendencias explícitas al momento de regular el uso del suelo, el agua y de este modo, la expansión agrícola. En este punto nos preguntamos si sería posible o económicamente viable el desarrollo de este modelo de producción del oasis de no poder, los nuevos actores, trasladar las externalidades.

De nuestro trabajo también se deriva la necesidad de entender que las particularidades hidro-geomorfológicas del valle, aquellas que lo hacen una zona expuesta a fenómenos aluvionales y que se constituyen en riesgos para el oasis producido, son a su vez explotadas como bienes y como servicios de forma exitosa por el nuevo modelo productivo. Esto es posible gracias a la disposición de elementos (capital) y capacidad de acción (poder de acción y transformación sobre el espacio) para trasladar los costos que de otro modo la geomorfología del lugar puede producir. En este punto se constata una gestión privada de los riesgos y las incertidumbres en las que algunos actores tienen la posibilidad de protegerse a sí mismos. Los nuevos actores del territorio se adaptan o vuelven resilientes a partir de una lógica privada en la que el mercado es el criterio prioritario y las capacidades de gestión del riesgo provistas por el estado están ausentes o subordinadas al primero. Esto se acerca a las reflexiones de Foucault sobre la adaptación, la seguridad y la gestión del riesgo como las formas hegemónicas contemporáneas en las que 
determinadas formas de vida constituyen la base del gobierno neoliberal (Foucault, 2008). En este mismo sentido, los desafíos de la adaptación a las incertidumbres del clima y la naturaleza invocan un nuevo sentido del homo economicus. Un tomador de decisiones autoorganizadas que se enfrentan a amenazas catastróficas, pero son capaces de realizar un manejo intencional de las contingencias. La capacidad no sólo es para prevenirlas, controlarlas y anularlas sino una gran capacidad para transferirlas.

\section{Referencias}

ADGER, W., ARNELL, N. \& TOMPKINS, E. Successful adaptation to climate change across scales, Global Environmental Change, 2005, Vol. 15, № 2, p. 77-86.

AMOORE, L. The Politics of Possibility: Risk and Security Beyond Probability. Durham: Duke University Press, 2013.

ATTERIDGE, A. \& REMLING, E. The Indirect Effects of Adaptation: Pathways for Vulnerability Redistribution in the Colombian Coffee Sector. Stockholm Environment Institute Working Paper, 2013, NNo10, p. 1- 40.

BARRIENTOS, J. Informe de Prospectiva Territorial Tunuyán 2030. Proyecto TCP/ARG/3302. Tupungato: INTA, IDR, Gobierno de Mendoza, 2013.

BONINSEGNA, J. Impacto del Cambio Climático en los Oasis del Oeste Argentino. Revista Ciencia e Investigación, CONICET DIGITAL, 2014, Tomo 64, № 1, p. 45-58 Disponible en Internet: http:// ri.conicet.gov.ar/handle/11336/4849

CAPITANELLI. R.G. Geomorfología, procesos y formas de modelado de los terrenos. Buenos Aires: CEYNE, 1992.

DGI. Balance Hídrico Tunuyán Superior. Mendoza: Departamento General de Irrigación, 2016.

EAKIN, H., WINKELS, A. \& SENDZIMIR, J. Nested vulnerability: exploring cross-scale linkages and vulnerability teleconnections in Mexican and Vietnamese coffee systems. Environmental Science \& Policy, 2009, Vol. 12, № 4, p. 398-412.

FOUCAULT, M. El nacimiento de la biopolítica. México: Fondo de Cultura Económica, 2008.

HINKELAMMERT, F. \& MORA JIMÉNEZ, H. Hacia una economía para la vida. Preludio a una reconstrucción de la economía. Proyecto Justicia y Vida: Bogotá, 2009.

HORKHEIMER, M. Crítica de la razón instrumental. Buenos Aires: Sur, 1969.

KLEIN, R. J. T., NICHOLLS, R. J., RAGOONADEN, S., CAPOBIANCO, M., ASTON, J.\& BUCKLEY, E. N. Technological Options for Adaptation to Climate Change in Coastal Zones. Journal of Coastal Research, 2001, Vol. 17 №3, p.531-43. 
KVALE, S. Interviews: An introduction to qualitative research interviewing. Thousand Oaks: Sage Publications, 1996.

LARSIMONT, R. Modelo de Agronegocio, Agua y Ruralidad en los oasis de Mendoza, 1990-2017: hacia una Ecología Política Territorial. Tesis Doctoral. Universidad de Buenos Aires, Buenos Aires, 2018.

LARSIMONT, R. CARBALLO, O. \& IVARS, J. Las papas de la globalización: el complejo agroindustrial papero en el Valle de Uco, Mendoza, Argentina. Revista Iberoamericana de Viticultura, Agroindustria y Ruralidad. Buenos Aires: Instituto de Estudios Avanzados, 2018, Vol. 5, №13, p.182-99.

MANZUR, T., \& CARBALLO, O. Aportes a la estrategia de convergencia metodológica. Análisis de tipologías de Explotaciones Agropecuarias a partir del Censo Nacional Agropecuario 2008. Actas del V Encuentro Latinoamericano de Metodología de las Ciencias Sociales, 2016. Disponible en Internet: http://elmecs.fahce.unlp.edu.ar/v-elmecs/actas-2016

MONTAÑA, E. Las disputas territoriales de una sociedad hídrica. Conflictos en torno al agua en Mendoza, Argentina. Revista Interamericana de Economía Ecológica, 2008, Vol. 9, p. 1 - 17.

MUSSETTA, P. , BARRIENTOS, J., FERRER, C., MASIOKAS, M., VILLALBA, R., DEIS, L. \& CAVAGNARO, B. Vulnerabilidad y cambio climático en Mendoza: brechas sociales, modelo de desarrollo y transformación del territorio agrícola. Revista Estudios Socioterritoriales, 2018, № 23, p. 11-26.

NAVARRETE, M. GÓMEZ, J. \& GALLOPÍN, G. Syndromes of sustainability of development for assessing the vulnerability of coupled human-environmental systems. The case of hydrometeorological disasters in Central America and the Caribbean . Global Environmental Change, 2007, №17, p. 207-217.

PORTELA, J., DEBANDI, G., MASTRANTONIO, L. \& AQUINDO, N. Atender a la biodiversidad permitiría incorporar la valoración de servicios ecosistémicos intra finca. Cuarto Congreso Internacional de Servicios Ecosistémicos en los Neotrópicos: de la investigación a la acción, Mar del Plata, 2015, p.1-5.

O'BRIEN, K. \& LEICHENKO, R. Double exposure: assessing the impacts of climate change within the context of economic globalization. Global environmental change, 2010, Vol. 10, №3, p. 221-232.

SANTOS, M. La naturaleza del espacio. Técnica y Tiempo. Razón y Emoción. Madrid: Ariel, 2000.

TAYLOR, M. The political ecology of climate change adaptation: livelihoods, agrarian change and the conflicts of development. Londres- Nueva York: Routledge, 2015.

TAYLOR, S. \& BOGDAN, R. Introducción a los métodos cualitativos de investigación. Barcelona: Paidós, 1992.

VAN DEN BOSCH, M. E. \& BOCCO, A., Dinámica intercensal de los sistemas de producción agropecuaria de la Provincia de Mendoza. Mendoza: INTA, 2016. 
VASILACHIS DE GIALDINO, I. La investigación cualitativa. En VASILACHIS DE GIALDINO, I. Estrategias de investigación cualitativa . Buenos Aires: Gedisa, 2013, p. 23-64.

WALLERSTEIN, I. La reestructuración capitalista y el sistema-mundo. Conferencia magistral en el $X X^{\circ}$ Congreso de la Asociación Latinoamericana de Sociología Política, México: 1995. Disponible en Internet: https://flacsoandes.edu.ec/web/imagesFTP/1265665449.La_reestructuracion_capitalista_y_el_sistema.pdf

WALLERSTEIN, I. Ecología y costos de producción capitalistas: No hay salida. Red de Ecología Social. Conceptos y Tendencias, 2004. Disponible en Internet: http://ecologiasocial.com/2004/06/ ecologia-y-costes-de-produccion-capitalistas-no-hay-salida/

WEBER, M. La ética protestante y el espíritu del capitalismo. México: Premiá editora, 1991.

WATTS, J. Now and Then.The origins of political ecology and the rebirth of adaptation as a form of thought. En PERREAULT, T., BRIDGE, G., MC CARTHY J. The Routledge Handbook of Political Ecology. Londres: Routledge, 2015, p. 19-50.

\section{Otras fuentes:}

Nota de prensa: "El futuro de la provincia está en el agua subterránea" en Unidiversidad Universidad de Cuyo, Mendoza 22 de enero de 2015 disponible en http://www.unidiversidad.com.ar/ el-futuro-de-la-provincia-esta-en-el-agua-subterranea

Registro de Uso de la Tierra. Dirección de Agricultura y Contingencias Climáticas. Gobierno de la Provincia de Mendoza. 
